\title{
mistr: A Computational Framework for Mixture and Composite Distributions
}

by Lukas Sablica and Kurt Hornik

\begin{abstract}
Finite mixtures and composite distributions allow to model the probabilistic representation of data with more generality than simple distributions and are useful to consider in a wide range of applications. The R package mistr provides an extensible computational framework for creating, transforming, and evaluating these models, together with multiple methods for their visualization and description. In this paper we present the main computational framework of the package and illustrate its application. In addition, we provide and show functions for data modeling using two specific composite distributions as well as a numerical example where a composite distribution is estimated to describe the log-returns of selected stocks.
\end{abstract}

\section{Introduction}

During the history of financial mathematics mankind has developed many useful theories how to describe financial markets. While the models in asset pricing are generally covered by the central limit theorem (CLT) arguments, these arguments do not cover the tail behaviour and thus are usually not appropriate in the risk management with its focus on the tails of the distribution. A simple illustration might be the log-returns distribution of the German multinational software corporation SAP. Clearly, the tails are much heavier than in the case of normal distribution with the same mean and standard deviation. This behavior can be frequently found in a number of financial assets.
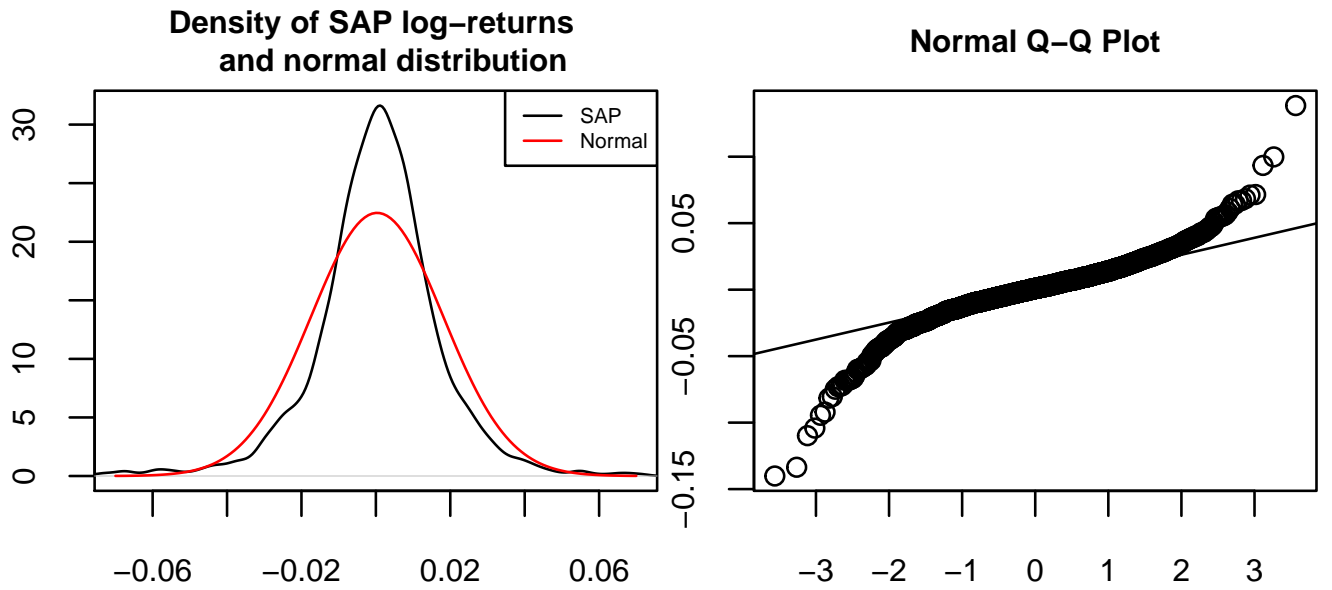

Figure 1: Left: Densities of the normal distribution and daily SAP log-returns from January 2007 to October 2017. Right: Quantile-quantile plot of the normal distribution against SAP log-returns.

A more interesting result can be seen from the quantile-quantile plot. While the normal distribution fails in the tails, it excels in the center. This suggests to use a more suitable distribution which can catch the fat tails presented above, yet follows a normal distribution in the center.

A simple answer to this idea is the concept of composite distributions (also known as spliced distributions) and mixture models, where one assumes that the distribution is a finite mixture of component distributions defined as

$$
F(x)=\sum_{i=1}^{n} w_{i} F_{i}\left(x \mid B_{i}\right), \quad f(x)=\sum_{i=1}^{n} \frac{w_{i}}{F_{i}\left(B_{i}\right)} 1_{B_{i}}(x) f_{i}(x)
$$

and

$$
F(x)=\sum_{i=1}^{n} w_{i} F_{i}(x), \quad f(x)=\sum_{i=1}^{n} w_{i} f_{i}(x),
$$

respectively, where $w_{1}, w_{2}, \ldots, w_{n}$ are positive weights that sum up to one, $B_{1}, B_{2}, \ldots, B_{n}$ are Borel sets giving a disjoint partition of the support, $1_{B_{i}}$ is the indicator function of a set $B_{i}$, and $F_{1}, F_{2}, \ldots, F_{n}$ are the probability distributions over $\mathbb{R}$ with $F_{i}\left(B_{i}\right)>0$ for all $i=1,2, \ldots, n$. Obviously, the composite models are a specific case of the mixture models, where the corresponding probability distribution functions are truncated to some disjoint support. For the presented data, a three-component composite 
model with the first and third component being some heavy-tailed distribution and its negative transform, respectively, is clearly something that might be appropriate. Note that this also suggests the need of a transformation framework that would be able to perform monotonic transformations on the random variables that represent these exceedance distributions. This innovation is motivated by the fact that even though most of the extreme value distributions belong to the location-scale family, without a decreasing transformation these distributions cannot be used for modeling of heavy left tails.

Moreover, composite models have gained a fair amount of attention in actuarial loss modeling. Most frequently one employs models composed of two components, where the first is based on the lognormal distribution and defined on the positive reals, and the second distribution is chosen according to the data set to model extreme measurements. Common choices for these tail-modeling distributions are, for instance, the generalized Pareto distribution or the Burr distribution. Such models have been proposed by various authors, for more details see Nadarajah and Bakar (2013), Cooray and Ananda (2005) and Scollnik (2007).

To offer a general framework for univariate distributions and for mixtures in general, package mistr is specifically designed to create such models, evaluate or even fit them. This article introduces mistr and illustrates with several examples how these distributions can be created and used.

\section{Distributions in $\mathbf{R}$}

R currently employs the naming convention [prefix][name], where [name] corresponds to the name of the desired distribution and [prefix] is one of ' $\mathrm{p}$ ', ' $\mathrm{d}$ ', ' $\mathrm{q}$ ' or ' $r$ ', indicating, respectively, the distribution, density and quantile functions, or random number generation. However, there are many of restrictions in this concept. What would be desirable is that one would be able to treat a distribution as a variable and so to be able to send it to a function or perform transformations on the random variable it represents.

Naturally, one way to do this is by using the object oriented system in R. To even improve this idea, one can use some favored dispatching mechanism, like S3 or S4, to let the computer decide how to handle the corresponding

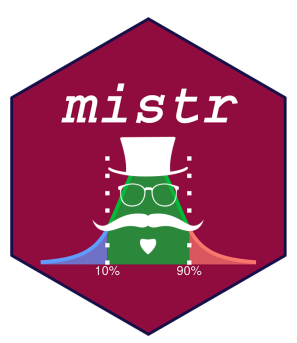
distribution correctly and which functions to use. In particular, the prefixes $p$, $\mathrm{d}, \mathrm{q}$, and $\mathrm{r}$ can still be just smartly evaluated as generic functions with appropriate methods. Moreover, with such a system we can add other useful calls and so take the distribution operations to the next level, such as monotonic transformation of a distribution. Additionally, once these objects containing all necessary information about the distributions are defined, they can be then reused for the purpose of the mixture and composite distributions.

This approach has already been used in the package distr (Ruckdeschel et al., 2006), which provides a conceptual treatment of distributions by means of S4 classes. A parent class Distribution allows to create objects and contains a slot for parameters as well as for the four methods mentioned above, $p(), d(), q()$, and $r()$. While distr provides several classes for distributions and finite mixtures in general, like many similar packages, to the best of our knowledge it does not contain any generating functions for the composite distributions. In particular, the only packages available for composite models are CompLognormal (Nadarajah and Bakar, 2013), evmix (Hu and Scarrott, 2018), OpVar (Zou et al., 2018), ReIns (Reynkens and Verbelen, 2018) and gendist (Bakar et al., 2016), which do not offer a general framework for composite models with more than two components. Whereas distr provides an impressive functionality-rich and extensible framework implemented in S4, we decided after careful deliberation to realize mistr based on a self-contained, light-weight framework using S3. This offers to adjust the representations and settings of the distributions exactly to the required needs by either mixture or composite models.

The framework provided by package mistr currently supports all distributions that are included in the stats package and, in addition, it offers some extreme value distributions like generalized Pareto, Pareto, Frechet, and Burr. In case that the user would like to use a distribution that is not directly supported by the framework, a new distribution can be implemented in a very simple way. This procedure is documented in detail in the Extensions vignette of the package.

As was already mentioned, the objects in $\mathrm{R}$ that represent a distribution need to contain all the necessary information. This is, of course, for the purpose of evaluating the distribution function or quantile function at suitable points just the knowledge of the parameters. Since the framework we propose is beyond a simple distribution evaluation, the representation is a little bit more complex. A simple random variable or a distribution is in our framework represented by the distribution family which the distribution follows, together with the proper parameters and some additional information that corresponds to the distribution. This information is either stored as a type of class or as attributes 
in a list. The framework then uses the S3 dispatching mechanism to use this information, and hence it works with the distribution. Such a representation allows to go beyond the simple $\mathrm{p}, \mathrm{d}, \mathrm{q}$, and $\mathrm{r}$ evaluation and enables to define new and more complicated functions.

An example is the left-hand limit of the cumulative distribution function. It might not look of crucial importance to be able to evaluate $F(x-)=\mathbb{P}(X<x)$, but this function plays a huge role in the transformations and composite distributions. Of course, this function differs from the standard distribution function only if it is applied to a distribution with a positive probability mass in the point of interest. For this reason we simply characterize the ("pseudo") support by the three parameters "from", "to" and "by". While the parameters "from" and "to" specify the range in which the distribution takes positive values, the parameter "by", which is only a part of the lattice-valued distributions, describes the deterministic step in the support and for most known discrete distributions is equal to one, since they have support just on the integers. It might of course differ for some distributions, which have support only on even numbers, or some scaled distributions. It is essential that this parametrization allows to perfectly define the support of a distribution, and hence allows to do more complicated operations and calculations, e.g., the left-hand limit of the distribution function. In the case the user would like a distribution with no equally distanced outcomes, one can perform a non-linear transformation, which will be dealt with in the next chapter.

\section{Combining distributions}

Having the framework defined for the simple distributions, it is desired to combine these objects in order to define a larger class of distributions. The framework currently supports the mixture and composite models given in, respectively, (2) and (1).

Following the definition of the composite models in the univariate case, the interval representation of the truncation allows to use a sequence of breakpoints

$$
-\infty=\beta_{0}<\beta_{1} \leq \beta_{2} \leq \cdots \leq \beta_{n-1}<\beta_{n}=\infty
$$

to fully characterize the partitions $B_{1}, B_{2}, \ldots, B_{n}$. Note that if $F_{i}$ is continuous, to ensure that the interval has positive probability we must take $\beta_{i-1}<\beta_{i}$.

This allows to define $\lambda_{1}=0$ and for all $i=2, \ldots, n$,

$$
\lambda_{i}=\left\{\begin{array}{cc}
F_{i}\left(\beta_{i-1}\right) & \text { if } \beta_{i-1} \notin B_{i}, \\
F_{i}\left(\beta_{i-1}\right) & \text { otherwise, }
\end{array}\right.
$$

where as before $F_{i}\left(\beta_{i-1}-\right)$ is the left-hand limit $\mathbb{P}\left(X_{i}<x\right)$, and for all $i=1,2, \ldots, n-1$,

$$
\rho_{i}=\left\{\begin{array}{cc}
F_{i}\left(\beta_{i}\right) & \text { if } \beta_{i} \in B_{i} \\
F_{i}\left(\beta_{i^{-}}\right) & \text {otherwise }
\end{array}\right.
$$

with $\rho_{n}=1$. Then for any $x \in B_{i}$

$$
F_{i}\left((-\infty, x] \cap B_{i}\right)=\left\{\begin{array}{cl}
F_{i}(x)-F_{i}\left(\beta_{i-1}\right) & \text { if } \beta_{i-1} \notin B_{i}, \\
F_{i}(x)-F_{i}\left(\beta_{i-1}\right) & \text { if } \beta_{i-1} \in B_{i} .
\end{array}\right.
$$

This means that for every $x \in B_{i}$ we can write the distribution as $F_{i}\left((-\infty, x] \cap B_{i}\right)=F_{i}(x)-\lambda_{i}$.

The straightforward implication of the above equations is that $\sup _{x \in B_{i}} F_{i}(x)=\rho_{i}$. Thus,

$$
F_{i}\left(B_{i}\right)=\rho_{i}-\lambda_{i}
$$

Hence, if we define $p_{i}=\sum_{j: j \leq i} w_{i}$ the composite distribution satisfies

$$
F(x)=p_{i-1}+w_{i} \frac{F_{i}(x)-\lambda_{i}}{F_{i}\left(B_{i}\right)}=p_{i-1}+w_{i} \frac{F_{i}(x)-\lambda_{i}}{\rho_{i}-\lambda_{i}}, \quad \forall x \in B_{i}
$$

and, in addition,

$$
p_{i-1} \leq F(x) \leq p_{i}, \quad \forall x \in B_{i} \quad \text { and } \quad \sup _{x \in B_{i}} F(x)=p_{i}
$$

If we take some $p \in(0,1)$, then since $\sum_{i} p_{i}=1$, there exists $i$ such that $p_{i-1}<p<p_{i}$ or $p=p_{i}$. From this it follows that

$$
F(x) \geq p \Leftrightarrow F_{i}(x) \geq \lambda_{i}+\frac{p-p_{i}}{w_{i}}\left(\rho_{i}-\lambda_{i}\right)
$$


which implies

$$
F^{-1}(p)=F_{i}^{-1}\left(\lambda_{i}+\frac{p-p_{i}}{w_{i}}\left(\rho_{i}-\lambda_{i}\right)\right), \text { for } p_{i-1}<p \leq p_{i}
$$

Using the quantile transformation method and the above quantile function we can easily simulate from our composite distribution.

Therefore, to fully specify a composite distribution, in addition to the mixture specifications, one needs to set the values that correspond to the breakpoints, which split $\mathbb{R}$ into disjoint partitions. Moreover, if at least one distribution is not absolutely continuous, it might be desired to specify to which adjacent interval should the breakpoint be included.

\section{Computational framework}

The objects representing a simple distribution can be created very easily. The creator functions follow a standard naming convention from $\mathrm{R}$ where the "dist" suffix is appended to the name of a distribution, and the parameters are entered as arguments. Thus, an object representing normal distribution with mean equal to 1 and standard deviation equal to 3 can be created as follows:

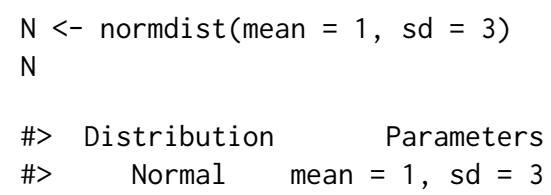

Once the objects are created, they can be used for evaluation of various functions. The most commonly employed functions clearly will be the methods for print () already demonstrated, and the methods for the functions $p(), d(), q()$ and $r()$. These can be easily evaluated as

$d(N, c(1,2,3))$

\#> [1] $0.1329808 \quad 0.1257944 \quad 0.1064827$

$p(N, c(1,2,3))$

\#> [1] $0.5000000 \quad 0.63055870 .7475075$

$q(N, c(0.1,0.2,0.3))$

\#> [1] $-2.8446547-1.5248637-0.5732015$

$r(N, 3)$

\#> [1] $\quad 1.8144430-2.6908086 \quad 0.2704776$

Additional important functions provided by mistr are plim() and qlim(), which implement, respectively, the already mentioned left-hand limit of the cumulative distribution function $F(x-)=$ $\mathbb{P}(X<x)$ and its pseudoinverse

$$
Q(p+)=\inf \{x \in \mathbb{R}: p<\mathbb{P}(X \leq x)\} .
$$

Note that the function qlim() plays a very important role when dealing with the transformations, and just as $\operatorname{plim}()$, in the case of continuous distributions it simplifies to $q()$. Clearly if $-X$ has a positive probability mass at $x$, then

$$
q=P(-X \leq x)=1-P(X<-x) \Rightarrow-Q_{X}(1-q+)=x .
$$

$\mathrm{B}<-$ binomdist $(\operatorname{size}=12$, prob $=0.3)$

$\operatorname{plim}(B, c(-3,0,3,12))$

\#> [1] $0.0000000 \quad 0.0000000 \quad 0.25281530 .9999995$

$q \lim (B, \operatorname{plim}(B, c(0,3,7,12)))$

\#> [1] $00 \begin{array}{llll}0 & 3 & 7 & 12\end{array}$ 


\section{Adding transformation}

Once the objects that represent a single distribution are created, we can use this representation to go beyond the scope of a simple distribution function evaluation. The knowledge of the distributions class and support range that is stored inside the object opens the doors for more complicated operations. One such an operation is the ability to perform monotone transformations of random variables.

The transformation framework currently allows for all standard monotone transformations like addition, subtraction, multiplication, division, logarithm, exponential and monotonic power transformations, where in case of binary operators a numeric value must be used as the second operand. Moreover, it is provided with the knowledge of invariant and direct transformations that correspond to the distributions it offers. This information is stored as a generic function that directly dispatches on the class of distribution family and not on the class univdist to prevent losing any information about the distribution. An example might be the exponential distribution where a multiplication with a positive scalar rather keeps the family and changes the parameters. On the other hand, a positive power transformation will directly create a Weibull distribution with appropriate parameters.

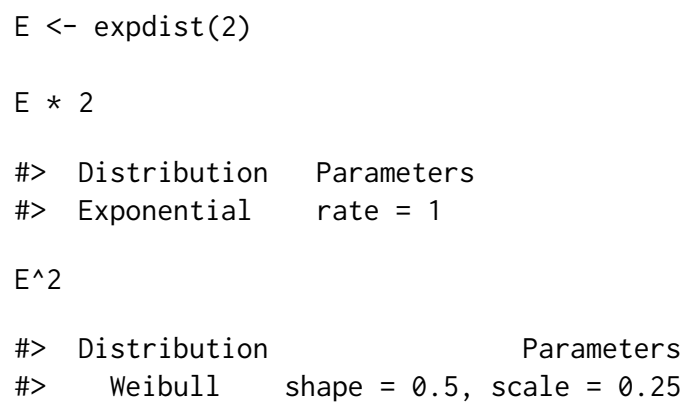

If the transformation is necessary and continuous on the support of the distribution, the transformation dispatches on the class univdist. For any untransformed distribution, this function will change the whole class and the class of the distribution family is removed since the random variable does not follow the distribution anymore. However, the information is stored for the case the distribution would need to untransform itself later. The function then builds an expression for the transformation and inverse transformation, along with a print expression and an expression for the derivative of the inverse transformation. Besides these list members, also a history member is stored, a list that records the information about the old transformations and becomes really handy when it comes to an inverse transformation of the previous one, or updating a transformation. A simple example of a transformation and update follows.

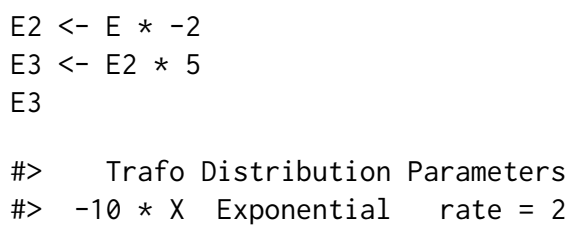

The next example uses the normal distribution that we created in the last chapter.

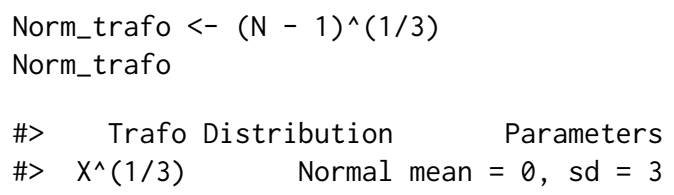

Note that the $X-1$ transformation is not displayed in the Trafo column as it is an invariant transformation that rather changed the parameter mean from 1 to 0 .

The methods that evaluate the transformed distribution are called in the same fashion as the nontransformed distributions. Additionally, the new pseudo description of the support can be returned using sudo_support(), which gives two numeric values ("From" and "To") describing the range of the support.

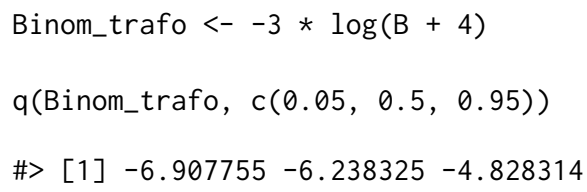


plim(Binom_trafo, $c(-6,-5,0))$

\#> [1] $0.5074842 \quad 0.91497501 .0000000$

sudo_support(Binom_trafo)

\#> From To

\#> $-8.317766-4.158883$

\section{Visualization}

In addition, the plot() and autoplot() generics can be called. These methods are offered for any distribution object in the mistr package and return the plot of PDF or PMF and CDF of a given object. The function uses the introduced $d()$ and $p()$ functions to evaluate the required values. While the plot () methods offer a plot constructed using base plotting, the autoplot () offers an alternative plot that is created using the ggplot2 package (Wickham, 2016).

$\operatorname{par}(\operatorname{mai}=c(0.4,0.4,0.2,0.2))$

plot(Norm_trafo, $x \lim 1=c(-2.5,2.5), y l a b 1=" n$, cex.axis $=0.75)$
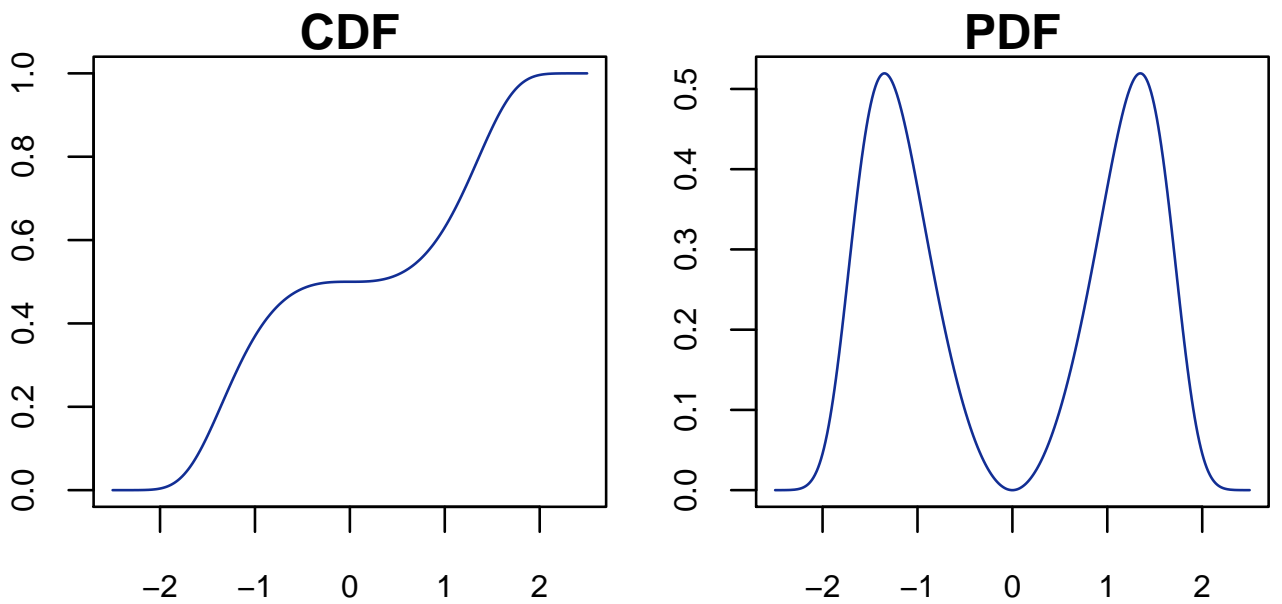

Figure 2: CDF (left) and PDF (right) plot of the Norm_trafo object using plot().

library (ggplot2)

autoplot(Norm_trafo, $x \lim 1=c(-2.5,2.5))$

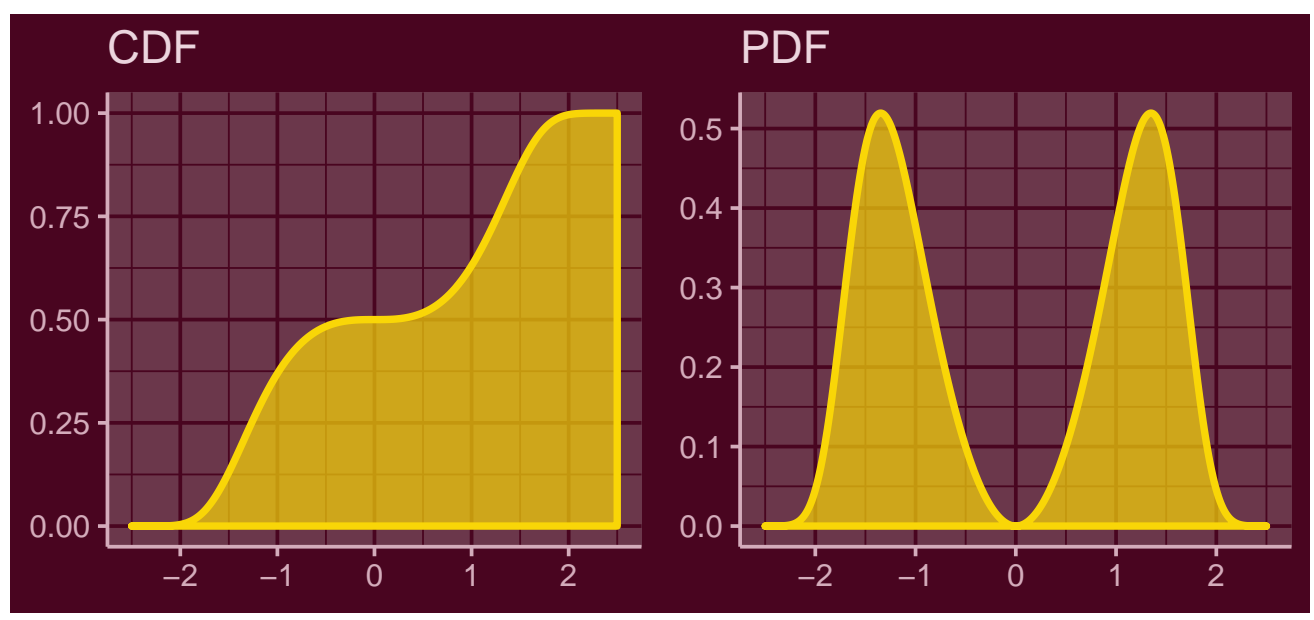

Figure 3: CDF (left) and PDF (right) plot of the Norm_trafo object using autoplot().

Other plot functions offered for the mistr distribution objects are QQplot() and QQplotgg(). The methods for this functions graphically compare the empirical quantiles of two data sets, or quantiles of two distribution objects, or quantiles of a distribution with the empirical quantiles of a sample. If 
quantiles of a continuous distribution are compared with a sample, a pointwise asymptotic confidence bound for this data is offered. This confidence "envelope" is based on the asymptotic results of the order statistics. For a distribution $F$ as the number of observations $n$ tends to infinity, the $p^{\text {th }}$ sample quantile is asymptotically distributed as

$$
X_{([n p])} \sim A N\left(F^{-1}(p), \frac{p(1-p)}{n\left[f\left(F^{-1}(p)\right)\right]^{2}}\right),
$$

where $f(x)$ and $F^{-1}(p)$ are the density function and quantile function associated with $F(x)$, respectively. More details can be found on the order statistics Wikipedia page or in Wilks (1948), for example. For alternative bounds see Almeida et al. (2018).

QQplotgg(Norm_trafo, $r($ Norm_trafo, 1000), conf $=0.99, \quad y l a b=$ NULL, $x l a b=$ NULL)

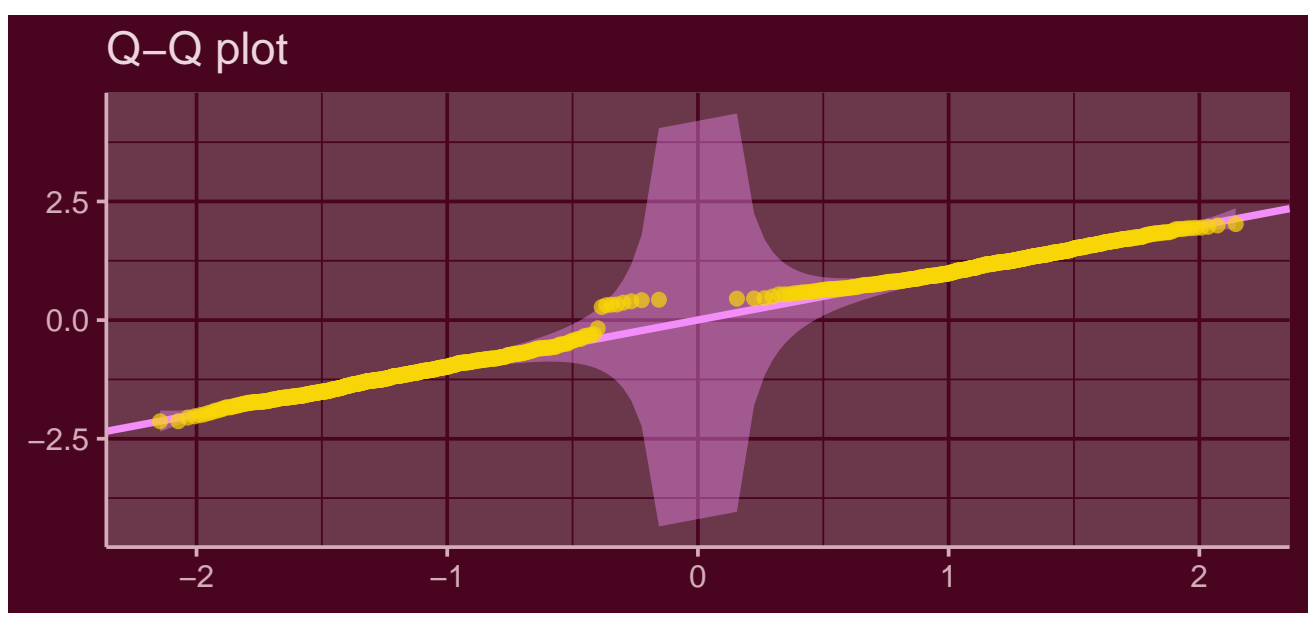

Figure 4: Q-Q plot of the Norm_trafo object against normally distributed sample with $99 \%$ confidence bound.

\section{Combining objects}

\section{Mixtures}

Mixture distributions are fully specified by the components $F_{i}(x)$ (i.e., the distributions) and by the weights $w_{i}$ that correspond to these components. Function mixdist () allows to create mixtures by specifying these characterizations. This can be done in two ways. First, the user may specify the distribution names (names from [prefix][name] functions), the list of appropriate parameters of these distributions, and a sequence of weights. An example of such a call follows.

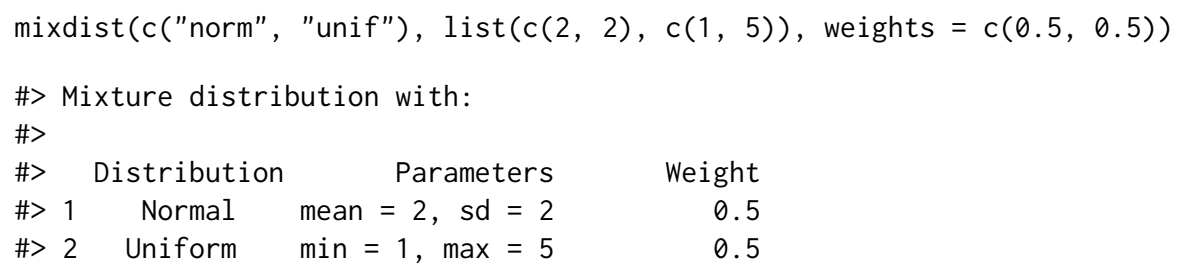

Another way is to use the objects that have already been defined. Since the parameters are already stored inside the object, all the function requires are the objects and the weights. This also allows to use transformed distributions from the last chapter or more complicated objects, which will be presented later. This means that the transformed normal and binomial distributions together with an exponential distribution can be reused for mixture distribution as:

$M<-$ mixdist(Norm_trafo, Binom_trafo, expdist( $(0.5)$, weights $=c(0.4,0.2,0.4))$

The information about the mixture can be accessed via multiple S3 methods. The components can be extracted using square brackets [], the weights can be obtained using weights(), and just as with standard distributions, the parameters are obtainable using parameters(). 
Other interesting methods are those for $q$ and qlim and mixdist objects. While finding the CDF and PDF of the mixture model is straightforward, an explicit general expression for quantile function of the mixture model is not available. However, it can be found numerically as a solution of a unit-root problem:

$$
\sum_{i=1}^{n} w_{i} F_{i}(Q(p))-p=0 .
$$

What is more, one can show that the quantile of a mixture distribution $Q(p)$ can always be found within the range of its components quantiles, and hence

$$
\min _{i \in\{1, \ldots, n\}} Q_{i}(p) \leq Q(p) \leq \max _{i \in\{1, \ldots, n\}} Q_{i}(p),
$$

where $Q_{i}(\cdot)$ is the quantile function of the $\mathrm{i}$-th component. This specifies the needed interval for the root finder that will then iteratively find the solution. Additionally, further problems are solved to return the correct values. To show how this algorithm works we perform a simple test and create a fully discrete mixture for which a decreasing transformation is applied. As the following plot reveals, all the values are calculated correctly.
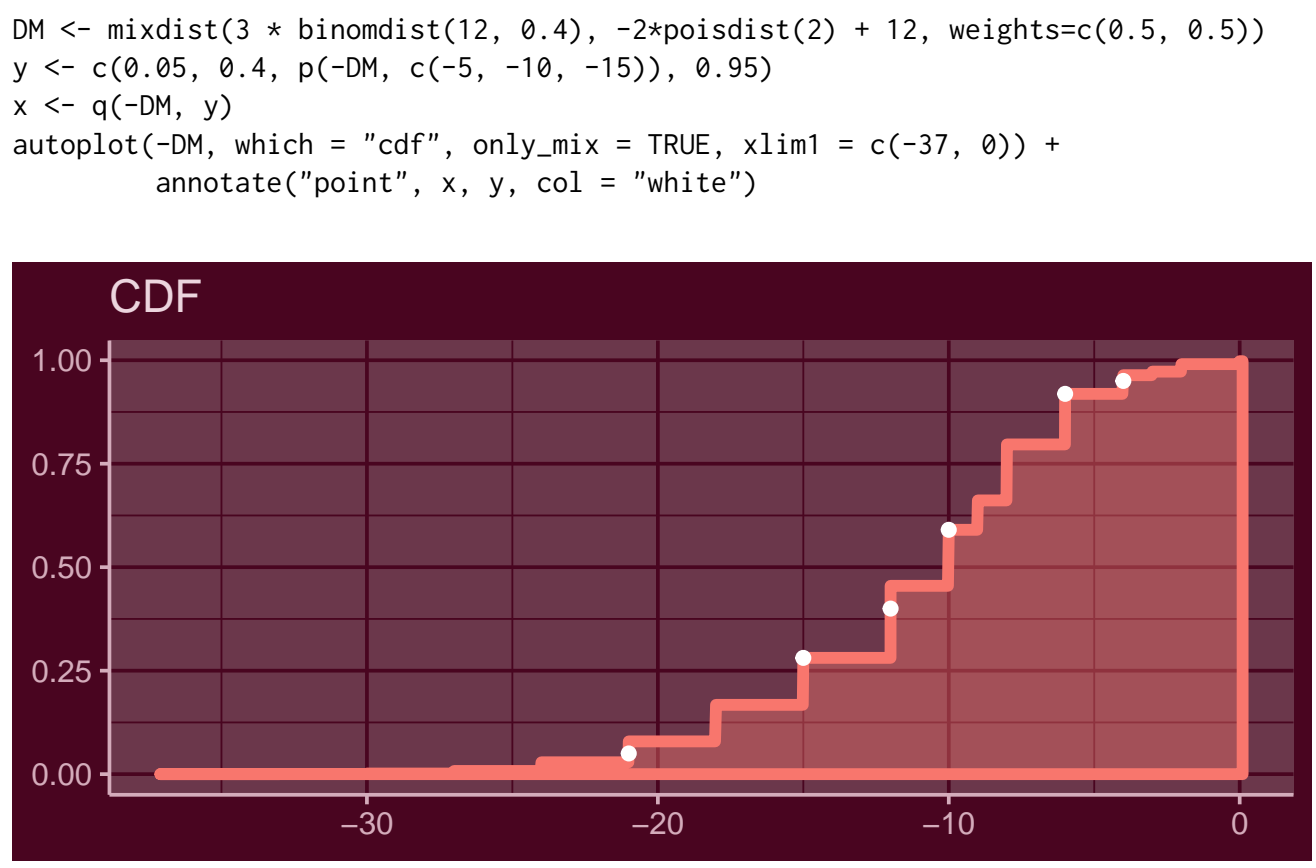

Figure 5: CDF plot of the -DM object with calculated quantiles annotated with white dots.

Finally, since inverse transform sampling is not efficient for mixture distributions, it can be replaced by first sampling according to the weights $w_{i}$ and then, conditionally on that, by drawing from the selected component, similarly as in the actuar package (Dutang et al., 2008). This approach is implemented in the corresponding method of the $r()$ function. This allows to draw from a mixture much faster than the inverse quantile transform method and can also be reused later for composite distributions. Besides the quantile function and other main functions for evaluation, one can call other generic functions that are designed for the class mixdist, e.g. sudo_support ().

sudo_support(M)

\#> From To

\#> -Inf Inf

Since the mixture models are in fact distributions, one can perform transformations of mixture random variables as well. It is easy to show that a transformation of a mixture random variable is the same as if we applied the same transformation to all of its components. In addition, since the support of the components is a subset of the mixture's support, if the framework allows to transform the mixture, then it does the components as well. Now using the mixture we created, we can perform a decreasing non-linear transformation. An example of $r()$ and autoplot () follows.

M_trans $<--2 *(M)^{\wedge}(1 / 3)$

r(M_trans, 4) 


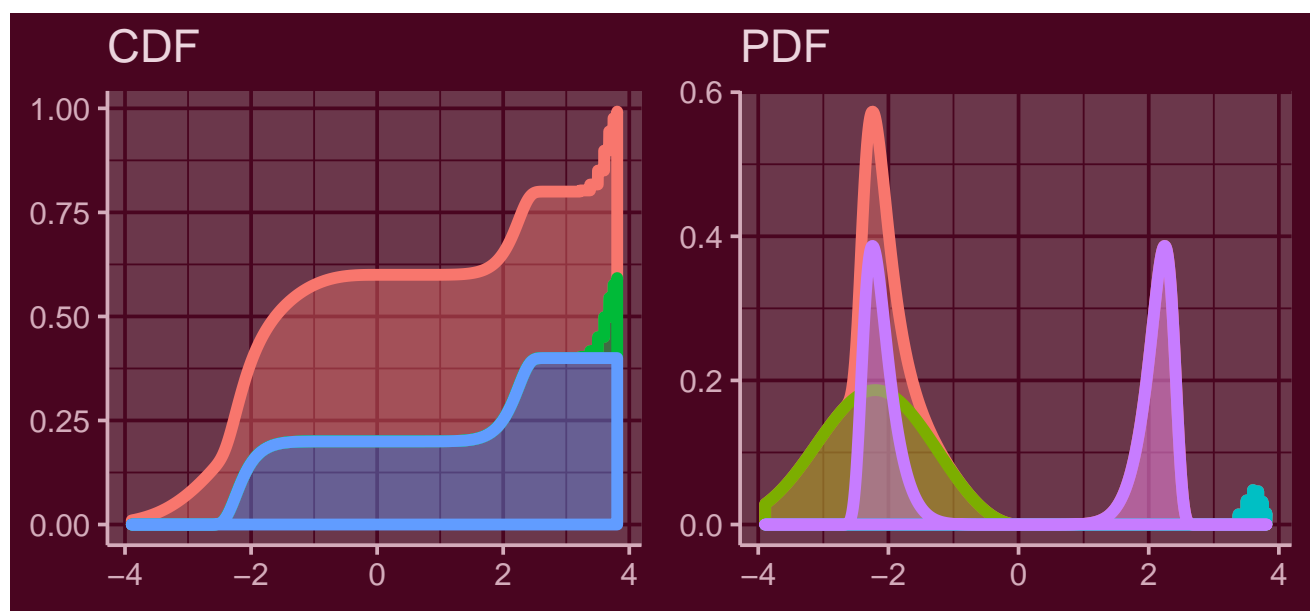

Figure 6: CDF (left) and PDF (right) plot of the M_trans object.

\section{Composite distributions}

Function compdist () creates composite distributions, which like mixture distributions can be done in two ways. Either one can directly use the objects or let the function create these objects by specifying the sequence of names and a list of parameters. In the following example, we will directly proceed with the first method where we define some objects inside the compdist () call to create a composite distribution. Besides these objects one needs to set the sequences of weights and breakpoints. Additionally, one may determine for each breakpoint to which partition should the breakpoint be included. This can be set by the argument break. spec with values ' $R$ ' or ' $L$ ', where ' $R$ ' and ' $L$ ' stand for right (i.e., include breakpoint to the interval on the right of the breakpoint) and left (i.e., include to the interval on the left), respectively. If this argument is not stated, the algorithm will by default set all intervals to be left-closed, i.e., right-open. This can be nicely seen from the following example where a linearly transformed Pareto distribution and a geometric distribution are combined with a normal distribution into a composite model.

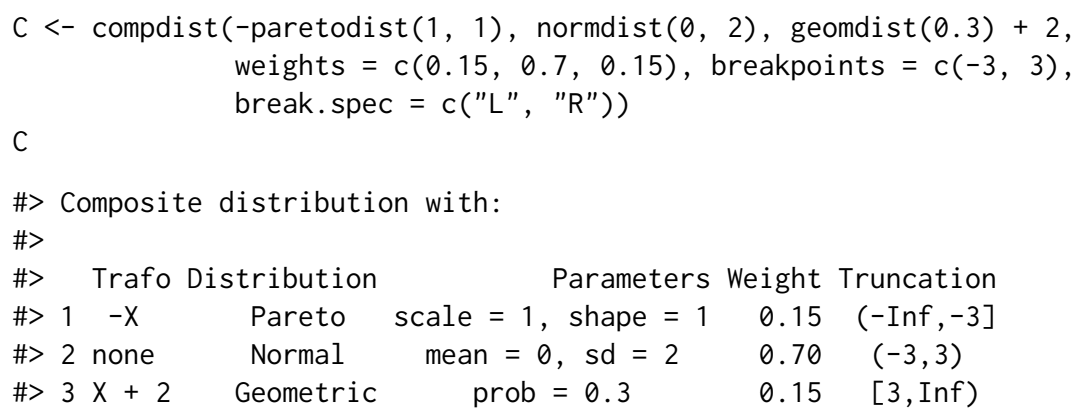

The argument break. spec is set to (" $L$ ", " $R$ "), and thus the breakpoint -3 belongs to the first partition while the second breakpoint is affiliated to the partition on the right. This can be observed from the print of the distribution, more precisely from the Truncation column, where the parentheses are printed according to this argument.

The package also permits to use the same breakpoint twice. This possibility allows to define a partition on a singleton, and hence to create a mass of probability. If this feature is used, the break.spec needs to be specified with " $R$ " and "L", for the first and second identical breakpoint, respectively, or not set at all. If the break. spec is not used, the source code will change break. spec such that this single point with probability mass is a closed set. This feature can become particularly useful when the user wants to create a distribution that is, for example, absolutely continuous on both the negative and positive reals and has positive mass at zero.

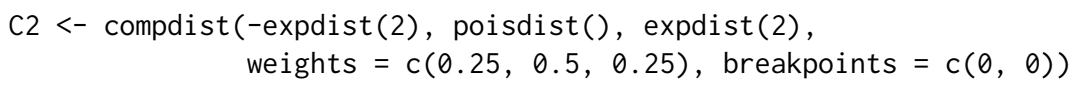




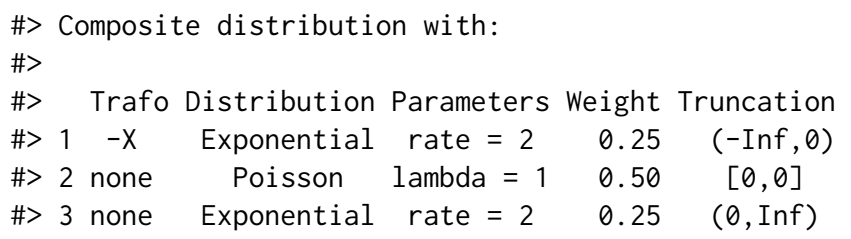

Note that the distribution assigned to this singleton has to be a discrete distribution with support on that point, otherwise the interval will have zero probability.

As for any distribution, the framework offers many methods that can be used to obtain additional information or evaluate the composite distribution. One can extract the parameters, weights, or the support in the same manner as with mixture distributions. In addition, calling breakpoints() extracts the splicing points. Finally, there are methods for plot() and autoplot () where the components are shown by default, which again can be turned off using the only_mix = TRUE argument.

$\operatorname{par}(\mathrm{mai}=\mathrm{c}(0.4,0.4,0.2,0.2))$

$\operatorname{plot}(C, x \lim 1=c(-15,15), y l a b 1=" \prime$, cex.axis $=0.75$, mtext_cex $=0.75)$
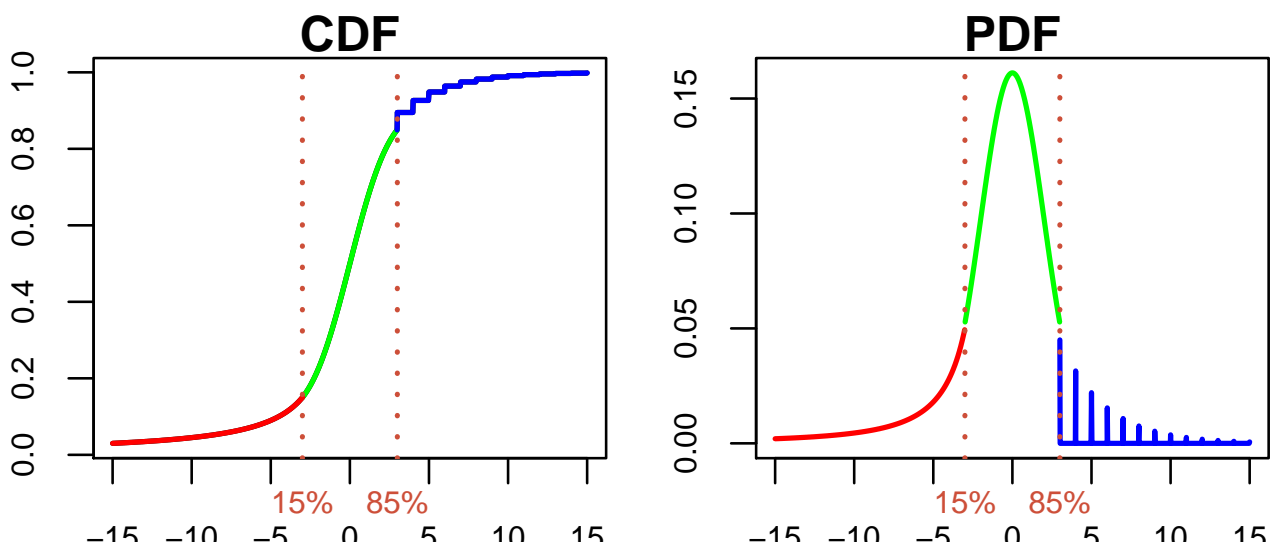

Figure 7: CDF (left) and PDF (right) plot of the $\mathrm{C}$ object using plot().

autoplot $(\mathrm{C} 2$, text_ylim $=0.01)$

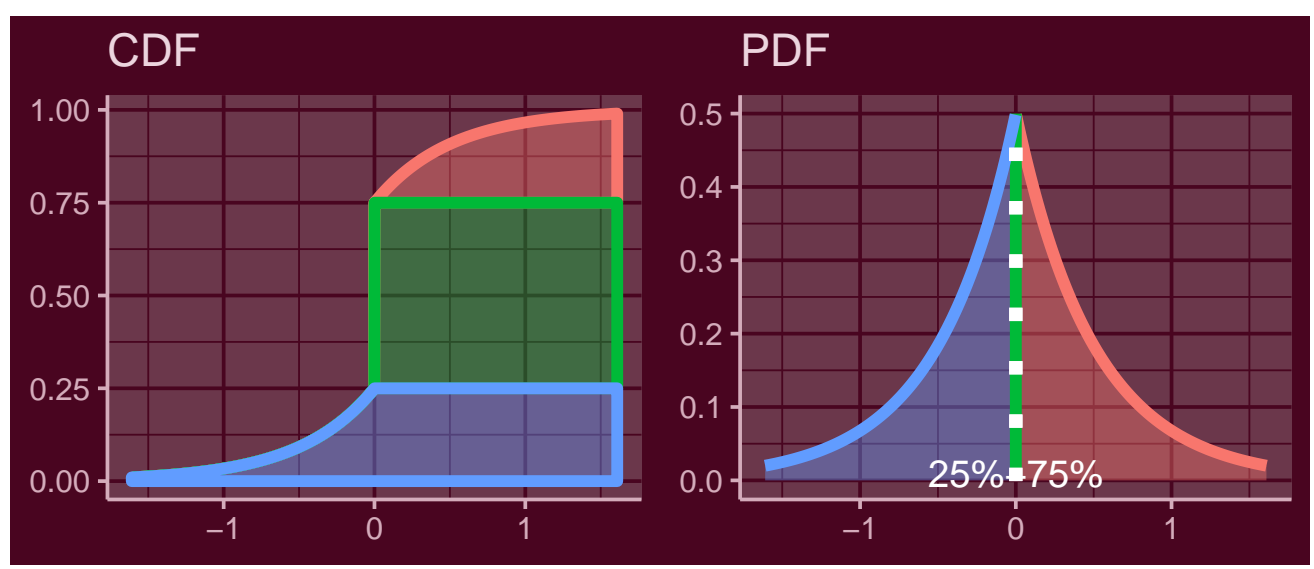

Figure 8: CDF (left) and PDF (right) plot of the C2 object using autoplot().

Analogously to the mixture distributions, the framework also supports the transformations of composite random variables. Thus, using the composite distribution we defined, we propose an example of a linear transformation.

C_trans $<--0.5 *(C+7)$

Even with such a distribution, the user still can evaluate all functions "of interest". To support this, an example follows where the function $q()$ and $r()$ are used, and the functions $p()$ and $d()$ are represented graphically using the autoplot() method. 

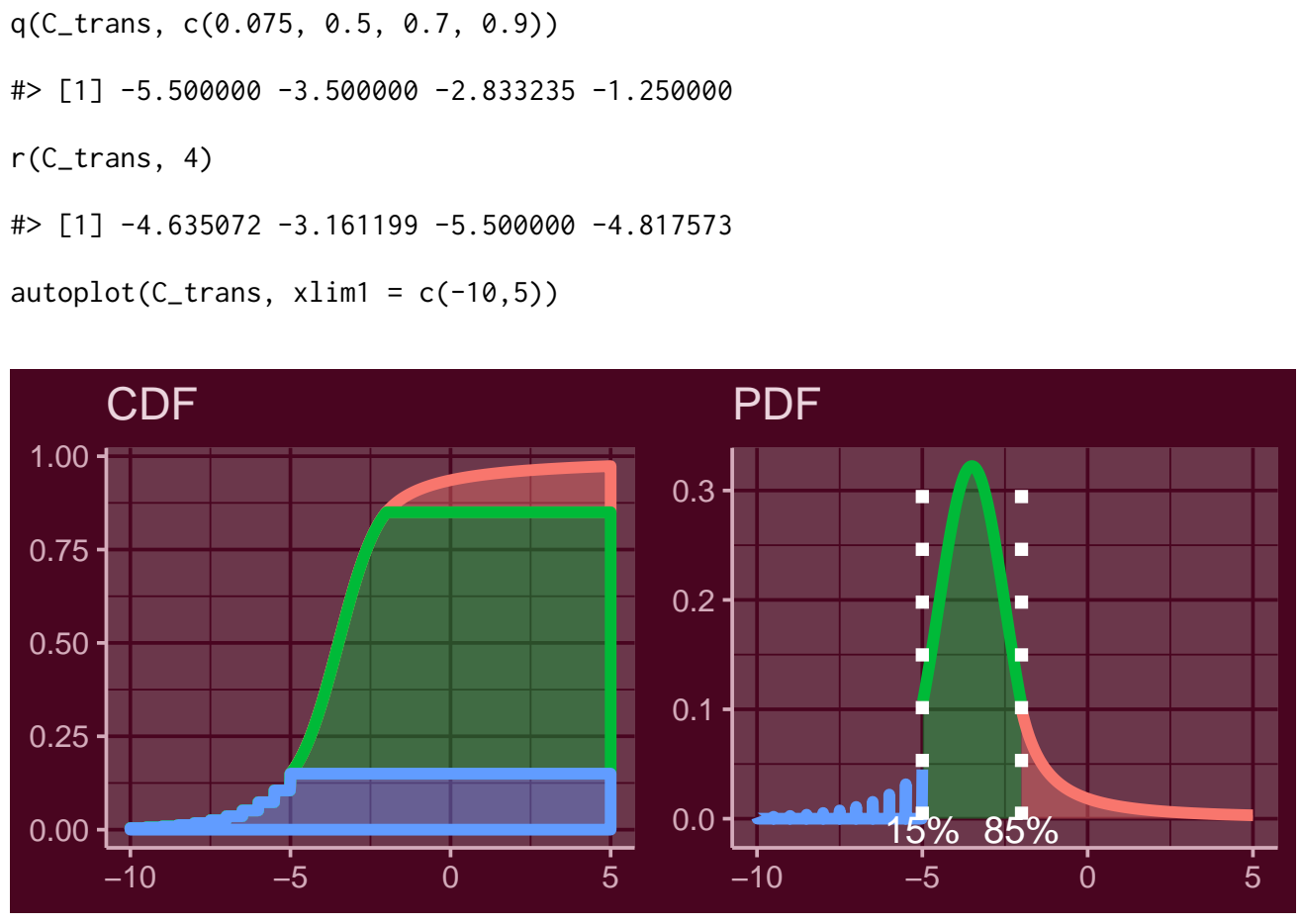

Figure 9: CDF (left) and PDF (right) plot of the C_trans object using autoplot().

\section{Combining mixture and composite distributions}

A significant advantage of object oriented programming is that the dispatching mechanism automatically knows how to treat a given object. This allows to combine mixture and composite models into more complicated mixtures and composite distributions. Therefore, we can take the transformed mixture and the transformed composite distribution that we created in the last chapter to compose a composite distribution with these distributions as components. What is more, we can perform further transformations of such a distribution.

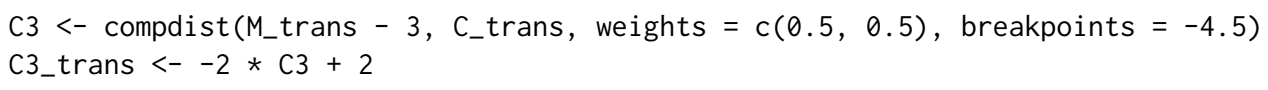

Thus, the object C3_trans is a transformed composite distribution that contains a transformed mixture and a transformed composite distribution, from which both additionally contain many transformed and untransformed distributions. Even in such complex models, the user may evaluate the most complicated functions like $p \lim ()$ and $q \lim ()$. The functions $d()$ and $p()$ can be again best represented graphically, where both distributions can easily be recognized from previous chapters.

autoplot(C3_trans, $x \lim 1=c(0,20)$, text_ylim $=0.01$, grey $=$ TRUE)

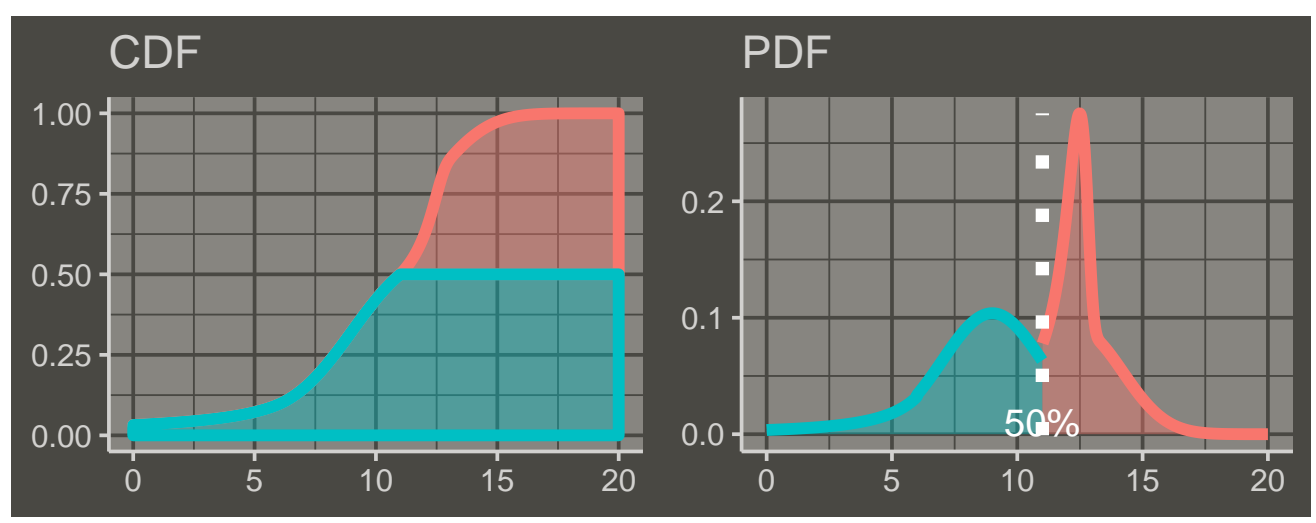

Figure 10: CDF (left) and PDF (right) plot of the C3_trans object. 
plim(C3_trans, c(6, 10, 12))

\#> [1] $0.09667553 \quad 0.421951890 .62458021$

qlim(C3_trans, $c(0.3,0.5,0.7))$

\#> [1] $\quad 8.785363 \quad 11.00000012 .327907$

As the print() output for such a hierarchical distribution does not contain lot of information, one can alternatively use summary () for which the package provides methods, which are particularly useful for the hierarchical distributions. The printed result of this call consists of all the necessary information, and much more as well. Nevertheless, since the result of summary() on the C3_trans object is two pages long, the demonstration is left to the reader.

To finish this chapter and to show that the user may go even further, we present an example where we combine the last object with another distribution from this chapter into a mixture distribution. The distribution is directly plotted using autoplot().

autoplot(mixdist $(c 3$ trans, $c 2+5$, weights $=c(0.7,0.3)), x \lim 1=c(0,15))$

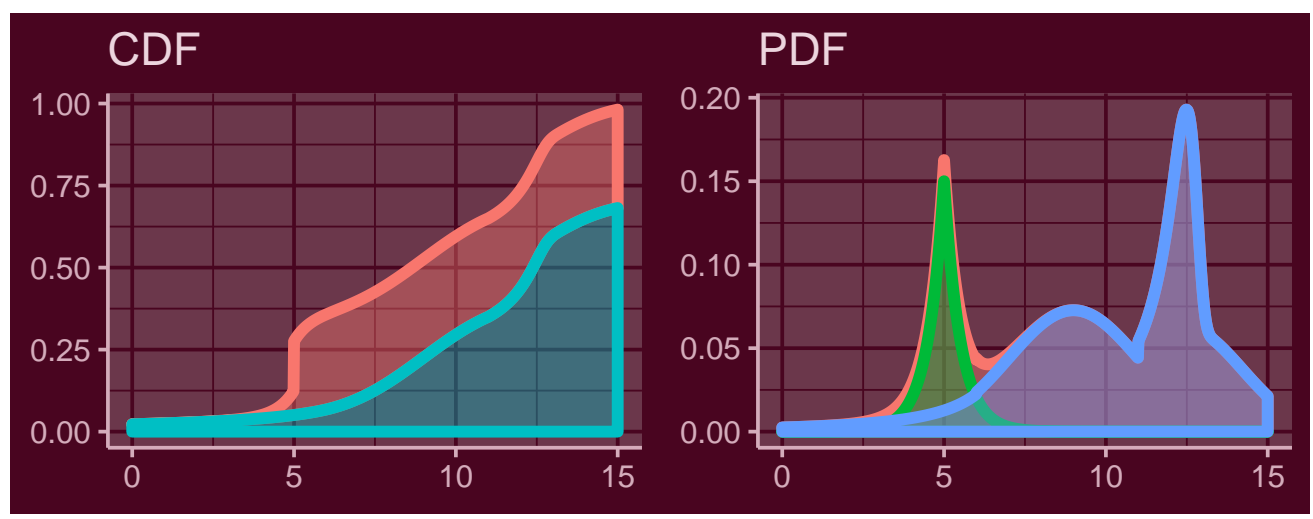

Figure 11: CDF (left) and PDF (right) plot of the mixture distribution with components C3_trans and C2 +5 .

\section{Data modeling}

While the previous chapters were aimed at showing the "muscles" (i.e., generality) of the framework, in this last chapter we will focus on examples using real data. In particular, we will present a simple fitting for two specific composite distributions.

As motivated in the introduction, the models in financial and actuarial mathematics suggest employing distributions which can capture the wide variety of behaviors in tails while still following the normal distribution in the center. In the two-tailed case, this suggests to use a three components composite distribution, where the first and third component will be used to model the extreme cases, i.e., the tails, and the second component will try to catch the center of the empirical distribution.

The first model offered by mistr is the Pareto-Normal-Pareto (PNP) model. This means that a $-X$ transformation of a Pareto random variable will be used for the left tail, normal distribution for the center and again Pareto for the right tail. From this it follows that the PDF of the model can be written as:

$$
f(x)=\left\{\begin{array}{ccc}
w_{1} \frac{f_{-P}(x)}{F_{-P}\left(\beta_{1}\right)} & \text { if } & -\infty<x<\beta_{1}, \\
w_{2} \frac{f_{N}(x)}{F_{N}\left(\beta_{2}\right)-F_{N}\left(\beta_{1}\right)} & \text { if } & \beta_{1} \leq x<\beta_{2}, \\
w_{3} \frac{f_{P}(x)}{1-F_{P}\left(\beta_{2}\right)} & \text { if } & \beta_{2} \leq x<\infty,
\end{array}\right.
$$

where $f_{P}(x)=f_{-P}(-x)$ and $F_{P}(x)=1-(K / x)^{\alpha}$ are the density and distribution function of a Pareto distribution with $F_{-P}(x)=1-F_{P}(-x) . f_{N}(x)$ and $F_{N}(x)$ are the PDF and CDF of the normal distribution, respectively.

If we follow the properties of a Pareto distribution, the conditional probability distribution of a Pareto-distributed random variable, given that the event is greater than or equal to $\gamma>K$, is again a Pareto distribution with parameters $\gamma$ and $\alpha$. This means that the conditional distribution $f_{P}(x \mid K, \alpha) /\left(1-F_{P}\left(\beta_{2} \mid K, \alpha\right)\right)=f_{P}\left(x \mid \beta_{2}, \alpha\right)$ if $\beta_{2}>K$. On the other hand, if $\beta_{2}<K$ the distribution 
cannot be continuous as the support of a Pareto distribution starts at $K$. The same can be shown for the transformed distribution and hence we can rewrite the PDF as

$$
f(x)=\left\{\begin{array}{ccc}
w_{1} f_{-P}\left(x \mid-\beta_{1}, \alpha_{1}\right) & \text { if } \quad-\infty<x<\beta_{1}, \\
w_{2} \frac{f_{N}(x \mid \mu, \sigma)}{F_{N}\left(\beta_{2} \mid \mu, \sigma\right)-F_{N}\left(\beta_{1} \mid \mu, \sigma\right)} & \text { if } \quad \beta_{1} \leq x<\beta_{2}, \\
w_{3} f_{P}\left(x \mid \beta_{2}, \alpha_{2}\right) & \text { if } \quad \beta_{2} \leq x<\infty
\end{array} \quad \text { where } \quad \begin{array}{c}
\beta_{1}<0<\beta_{2}, \\
\alpha_{1}, \alpha_{2}>0 .
\end{array}\right.
$$

The condition $\beta_{1}<0<\beta_{2}$ follows from the fact that the scale parameter has to be positive. Thus, such a model can be fully used only with demeaned data sample or with data with a mean close to zero. This is of course not a problem for stock returns, which are the aim of this chapter. What is more, one can show that the density is continuous if for the shape parameters it holds that

$$
\begin{gathered}
\alpha_{1}=-\beta_{1} \frac{w_{2} f_{N}\left(\beta_{1} \mid \mu, \sigma\right)}{w_{1}\left(F_{N}\left(\beta_{2} \mid \mu, \sigma\right)-F_{N}\left(\beta_{1} \mid \mu, \sigma\right)\right)}, \\
\alpha_{2}=\beta_{2} \frac{w_{2} f_{N}\left(\beta_{2} \mid \mu, \sigma\right)}{w_{3}\left(F_{N}\left(\beta_{2} \mid \mu, \sigma\right)-F_{N}\left(\beta_{1} \mid \mu, \sigma\right)\right)} .
\end{gathered}
$$

This condition is not only natural when modelling the real data, it additionally solves the possible instability effects at estimation once observations come to lie close to breakpoints. Due to the fact that a composite distribution can be represented as a mixture of truncated distributions that are truncated to a disjoint support, the weight of each component can be estimated as the proportion of points that correspond to each of the truncated regions. Obviously, this condition ensures that the empirical and estimated CDF match on each of the breakpoints. Thus, conditionally on the fact that the breakpoints are known, similarly as in Reynkens et al. (2017), the weights can be computed as

$$
w_{1}=\frac{\sum_{i=1}^{n} 1_{\left\{x_{i}<\beta_{1}\right\}}}{n}, w_{2}=\frac{\sum_{i=1}^{n} 1_{\left\{\beta_{1} \leq x_{i}<\beta_{2}\right\}}}{n}, w_{3}=\frac{\sum_{i=1}^{n} 1_{\left\{\beta_{2} \leq x_{i}\right\}}}{n},
$$

where $1_{\{\cdot\}}$ is the indicator function and $x_{i}$ is the $\mathrm{i}$-th data value. These conditions decrease the number of parameters from 11 to 4 and imply the density function of the form:

$$
f\left(x \mid \beta_{1}, \beta_{2}, \mu, \sigma\right) \text {. }
$$

This model is offered by the function PNP_fit() which takes the data and a named vector of starting values with names break1, break2, mean, and sd and returns a list of class comp_fit. Other arguments are passed to the optimizer which then maximizes the likelihood of the above specified model. The optimization is handled by the mle2() function from bbmle package (Ben Bolker and $\mathrm{R}$ Development Core Team, 2017). To demonstrate this, we will take the same data we used in the introduction to fit a PNP model with the default starting values.

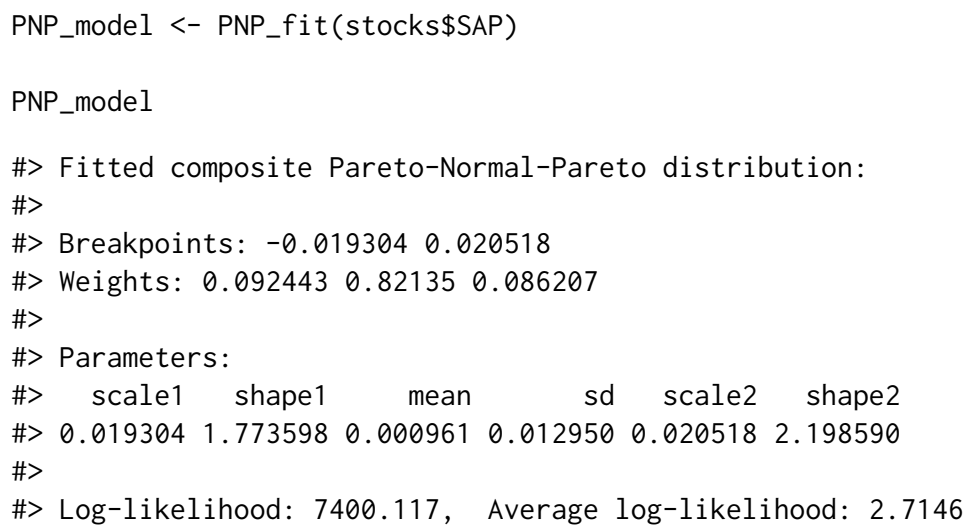

If the fitted object is printed, the function prints all the parameters together with the log-likelihood that was achieved by the optimization. In addition, the average log-likelihood is printed, which is just the log-likelihood divided by the size of the data set. The user can extract parameters using parameters(), weights using weights(), and breakpoints using breakpoints(). The distribution() function can be used to extract the distribution with fitted parameters that can be used for evaluation.

Finally, again plot() and autoplot() methods are offered, which give a Q-Q plot of the fitted distribution and the data, and the CDF and PDF plot of the fitted distribution, which overlap with the empirical CDF and PDF of the data set. Again, the which argument can extract the proposed plots separately (i.e., which = "pdf"). Other arguments are passed to the the plot calls.

plot (PNP_model, ylab1 ="", ylab2 ="") 


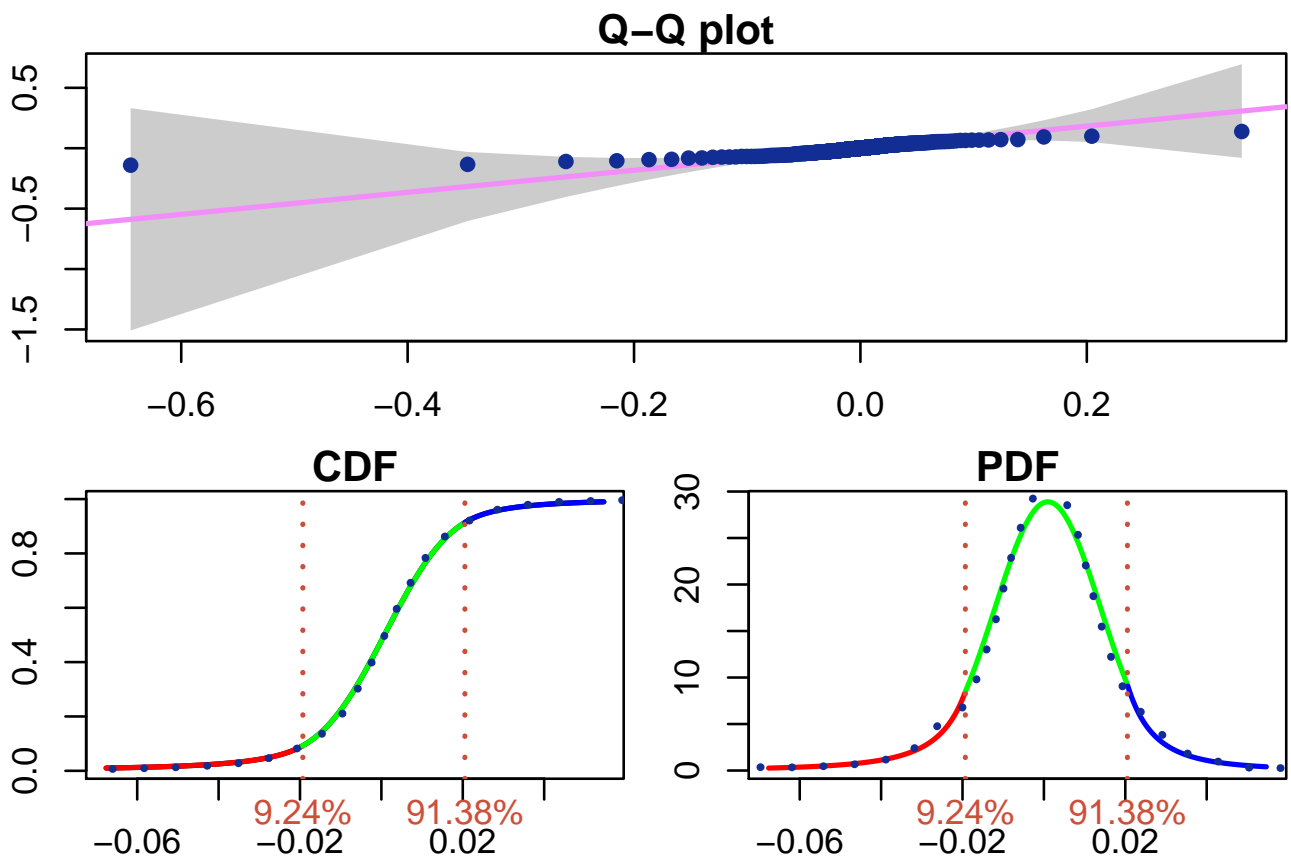

Figure 12: Q-Q (up), CDF (bottom left) and PDF (bottom right) plot of the fitted PNP model overlapped with empirical results indicated by blue dots using plot().

The plots indicate an overall nice fit where all quantiles are in the confidence bound.

The second model offered is a similar distribution to the previous one, except we will replace the Pareto distributions by the generalized Pareto distributions (GPD)

$$
F_{G P D}(x)=\left\{\begin{array}{cl}
1-\left(1+\xi \frac{x-\theta}{\gamma}\right)^{-1 / \xi} & \text { if } \xi \neq 0 \\
1-\exp \left(-\frac{x-\theta}{\gamma}\right) & \text { if } \xi=0
\end{array}\right.
$$

This means that the PDF of this model can be written as:

$$
f(x)=\left\{\begin{array}{ccc}
w_{1} \frac{f_{-G P D}(x)}{F_{-G P D}\left(\beta_{1}\right)} & \text { if } & -\infty<x<\beta_{1}, \\
w_{2} \frac{f_{N}(x)}{F_{N}\left(\beta_{2}\right)-F_{N}\left(\beta_{1}\right)} & \text { if } & \beta_{1} \leq x<\beta_{2}, \\
w_{3} \frac{f_{G P D}(x)}{1-F_{G P D}\left(\beta_{2}\right)} & \text { if } & \beta_{2} \leq x<\infty .
\end{array}\right.
$$

The same way as in the PNP model, the scale parameters can be eliminated by the continuity conditions, weights by the above mentioned condition and in addition, under current settings and the continuity conditions, the value of the conditional GPD distribution depends on the location parameter only through the conditions $-\beta_{1} \geq \theta_{1}$ and $\beta_{2} \geq \theta_{2}$. This suggests to choose without any loss in the model $-\beta_{1}=\theta_{1}$ and $\beta_{2}=\theta_{2}$. This PDF is then fully characterized by

$$
f\left(x \mid \beta_{1}, \beta_{2}, \mu, \sigma, \xi_{1}, \xi_{2}\right),
$$

where the only restriction on the parameters is $-\infty<\beta_{1}<\beta_{2}<\infty$.

These conditions decrease the number of parameters from 13 to 6 . What is more, the function GNG_fit() contains the argument break_fix, which fixes the breakpoints from the vector of starting values, and so decreases the number of parameters to 4 if TRUE is assigned. In this case, the breakpoints are fixed and weights are computed before the optimization. The function GNG_fit() takes the data, the named vector of starting values with names break1, break2, mean, sd, shape1 and shape2, the break_fix argument and the argument midd, which is by default set to be equal to the mean of the data. The midd value is used to split $\mathbb{R}$ into two sub-intervals and then the first breakpoint is optimized on the left of the midd value and the second breakpoint on the right.

The function returns a list of class comp_fit. The results can be then extracted, printed or visualized in the same way as the results of PNP_fit().

GNG_model <- GNG_fit (stocks\$SAP)

GNG_model 
\#> Fitted composite GPD-Normal-GPD distribution:

$\#>$

\#> Breakpoints: -0.0194140 .019353$

\#> Weights: 0.0913430 .8125460 .096112

$\#>$

\#> Parameters:

\#> loc1 scale1 shape1 mean sd loc2 scale2 shape2

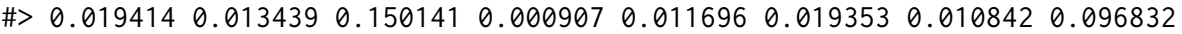

\#>

\#> Log-likelihood: 7423.245, Average log-likelihood: 2.7231

autoplot(GNG_model)

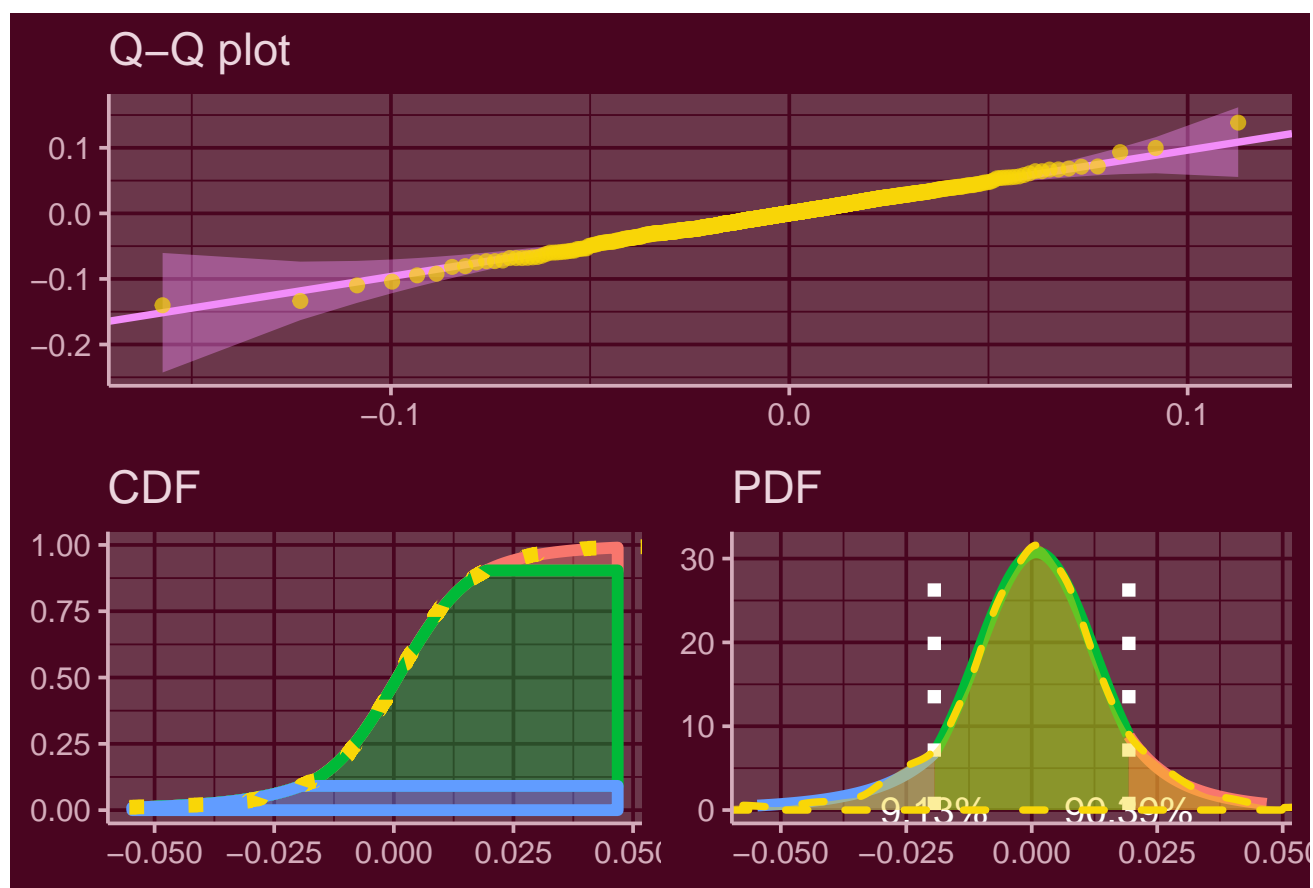

Figure 13: Q-Q (up), CDF (bottom left) and PDF (bottom right) plot of the fitted GNG model overlapped with empirical results indicated by yellow dots and dashes using autoplot().

The log-likelihood has increased to 7423.2 with the average of 2.723 per data-point. In this model, the generalized Pareto distribution explains the first 9.1\% from the left tail and the last $9.6 \%$ from the right tail. Since the GPD generalizes the Pareto distribution, the higher likelihood is a reasonable result. Moreover, the QQ-plot suggests an almost perfect fit.

The result of these estimations is a proper continuous parametric set-up that describes the distribution of the data. What is more, the distribution has been fitted as a whole with respect to the continuity conditions. This means that the tails take into account the whole distribution, which allows to calculate the risk measures with an even higher precision as when only the tails are modeled.

\section{Risk measures}

Package mistr provides a function risk() which can be used for rapid calculations of point estimates of prescribed quantiles, expected shortfalls and expectiles (in the following table denoted as VaR, ES and Exp, respectively). For more details on these measures see McNeil et al. (2015). As input parameters this function needs the output of the function PNP_fit() or GNG_fit() and a vector of the desired levels. As an example we illustrate this function on our fitted object.

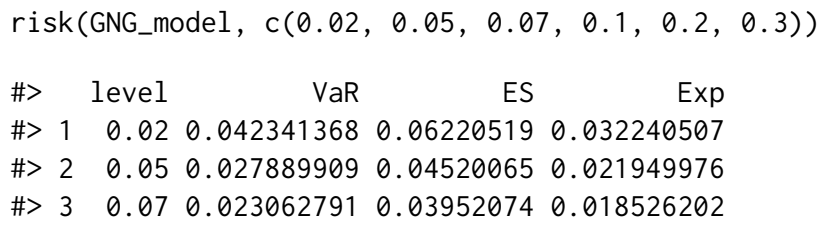


$\begin{array}{llllll}\text { \#> } 4 & 0.10 & 0.018245509 & 0.03380624 & 0.015091608\end{array}$

$\begin{array}{llllll}\#> & 5 & 0.20 & 0.010642738 & 0.02386181 & 0.008851640\end{array}$

\#> $6 \quad 0.30 \quad 0.006167236 \quad 0.01867190 \quad 0.005168659$

These results can be also visualized if arguments plot or ggplot are set to TRUE.

\section{Summary}

We introduced a new extensible framework for mixture and composite distributions in $\mathrm{R}$ via the mistr package. It offers creation and manipulation of simple distributions which can be combined into more complicated distributions to offer more generality in the current data modeling. Furthermore, the framework provides multiple methods specifically designed to describe and visualize the distributions or functions capable of fitting the two pre-defined composite distributions introduced in the last chapter.

The package is additionally equipped with the possibility to extend the current list of known distributions and monotone transformations by letting the user add these in a very simple way. This procedure is documented in detail in the Extensions vignette. Finally, we will keep adding multiple extensions and distributions to extend the generality even more.

\section{Bibliography}

A. Almeida, A. Loy, and H. Hofmann. ggplot2 Compatible Quantile-Quantile Plots in R. The R Journal, 10(2):248-261, 2018. URL https://doi .org/10.32614/RJ-2018-051. [p7]

S. Bakar, S. Nadarajah, Z. Kamarul Adzhar, and I. Mohamed. Gendist: An r package for generated probability distribution models. P L o S One, 11(6), 6 2016. ISSN 1932-6203. URL https: //doi .org/ 10.1371/journal. pone.0156537. [p2]

Ben Bolker and R Development Core Team. bbmle: Tools for General Maximum Likelihood Estimation, 2017. URL https://CRAN. R-project. org/package=bbmle. R package version 1.0.20. [p13]

K. Cooray and M. M. Ananda. Modeling actuarial data with a composite lognormal-pareto model. Scandinavian Actuarial Journal, 2005(5):321-334, 2005. URL https://doi.org/10.1080/ 03461230510009763. [p2]

C. Dutang, V. Goulet, and M. Pigeon. actuar: An r package for actuarial science. Journal of Statistical Software, Articles, 25(7):1-37, 2008. ISSN 1548-7660. URL https: //doi . org/10.18637/jss . v025. i07. [p8]

Y. Hu and C. Scarrott. evmix: An r package for extreme value mixture modeling, threshold estimation and boundary corrected kernel density estimation. Journal of Statistical Software, Articles, 84(5):1-27, 2018. ISSN 1548-7660. URL https://doi.org/10.18637/jss.v084.i05. [p2]

A. J. McNeil, R. Frey, and P. Embrechts. Quantitative Risk Management: Concepts, Techniques and Tools. Princeton University Press, Princeton, NJ, USA, 2015. ISBN 0691166277, 9780691166278. [p15]

S. Nadarajah and S. A. A. Bakar. CompLognormal: An R Package for Composite Lognormal Distributions. The R Journal, 5(2):97-103, 2013. URL https: //doi .org/10.32614/RJ-2013-030. [p2]

T. Reynkens and R. Verbelen. ReIns: Functions from "Reinsurance: Actuarial and Statistical Aspects", 2018. URL https://CRAN.R-project.org/package=ReIns. R package version 1.0.8. [p2]

T. Reynkens, R. Verbelen, J. Beirlant, and K. Antonio. Modelling censored losses using splicing: A global fit strategy with mixed erlang and extreme value distributions. Insurance: Mathematics and Economics, 77:65-77, 2017. URL https://doi .org/10.1016/j. insmatheco.2017.08.005. [p13]

P. Ruckdeschel, M. Kohl, T. Stabla, and F. Camphausen. S4 classes for distributions. R News, 6(2):2-6, May 2006. [p2]

D. P. M. Scollnik. On composite lognormal-pareto models. Scandinavian Actuarial Journal, 2007(1): 20-33, 2007. URL https://doi.org/10.1080/03461230601110447. [p2]

H. Wickham. ggplot2: Elegant Graphics for Data Analysis. Springer-Verlag New York, 2016. ISBN 978-3-319-24277-4. URL https: //ggplot2. tidyverse.org. [p6]

S. S. Wilks. Order statistics. Bulletin of the American Mathematical Society, 54(1):6-50, 1948. [p7] 
C. Zou, M. Pfeuffer, M. Fischer, K. Dehler, N. Derfuss, B. Graswald, L. Moestel, J. Wang, and L. Wicht. OpVaR: Statistical Methods for Modeling Operational Risk, 2018. URL https://CRAN. R-project. org/ package=0pVaR. R package version 1.0.5. [p2]

Lukas Sablica

Institute for Statistics and Mathematics

Vienna University of Economics and Business

Austria

https://www.wu.ac.at/en/statmath

ORCiD: 0000-0001-9166-4563

lsablica@wu.ac.at

Kurt Hornik

Institute for Statistics and Mathematics

Vienna University of Economics and Business

Austria

https://www.wu.ac.at/en/statmath

ORCiD: 0000-0003-4198-9911

Kurt.Hornik@wu.ac.at 